\title{
Thermal Hall signatures of non-Kitaev spin liquids in honeycomb Kitaev materials
}

\author{
Yong Hao Gao, ${ }^{1}$ Ciarán Hickey, ${ }^{2}$ Tao Xiang, ${ }^{3,4}$ Simon Trebst, ${ }^{2}$ and Gang Chen $\odot^{1,5}$ \\ ${ }^{1}$ State Key Laboratory of Surface Physics and Department of Physics, Fudan University, Shanghai 200433, China \\ ${ }^{2}$ Institute for Theoretical Physics, University of Cologne, 50937 Cologne, Germany \\ ${ }^{3}$ Institute of Physics, Chinese Academy of Sciences, Beijing 100190, China \\ ${ }^{4}$ Department of Physics, University of Chinese Academy of Sciences, Beijing 100049, China \\ ${ }^{5}$ Department of Physics and Center of Theoretical and Computational Physics, University of Hong Kong, \\ Pokfulam Road, Hong Kong, China
}

(Received 7 June 2019; revised manuscript received 2 August 2019; published 26 August 2019)

\begin{abstract}
Motivated by the recent surge of field-driven phenomena discussed for Kitaev materials, in particular the experimental observation of a finite thermal Hall effect and theoretical proposals for the emergence of additional spin liquid phases beyond the conventional Kitaev spin liquid, we develop a theoretical understanding of the thermal Hall effect in honeycomb Kitaev materials in magnetic fields. Our focus is on gapless U(1) spin liquids with a spinon Fermi surface, which have been shown to arise as field-induced phases. We demonstrate that in the presence of symmetry-allowed second-neighbor Dzyaloshinskii-Moriya interactions these spin liquids give rise to a finite, nonquantized, thermal Hall conductance in a magnetic field. The microscopic origin of this thermal Hall effect can be traced back to an interplay of Dzyaloshinskii-Moriya interaction and Zeeman coupling, which generates an internal U(1) gauge flux that twists the motion of the emergent spinons. We argue that such a nonquantized thermal Hall effect is a generic response in Kitaev models for a range of couplings.
\end{abstract}

DOI: 10.1103/PhysRevResearch.1.013014

\section{INTRODUCTION}

The first experimental observation of a quantum Hall effect in two-dimensional electron systems [1] has proved to be a scientific revolution, with its exact quantization of Hall resistance raising measurement standards to unprecedented levels of precision [2]. It has also served as a blueprint for the interplay between experimental breakthroughs and deep conceptual progress on the theoretical side. For the integer quantum Hall effect, it was the seminal introduction of topological invariants [3] that explained the quantization of conductance. For the subsequent fractional quantum Hall effect [4], it was the theoretical concepts of emergence and fractionalization [5]. The observation of the quantum spin Hall effect [6] marked the birth of the topological insulator [7]. Therefore, the more recent observation of a half-integer quantized thermal Hall effect [8,9] has caught the imagination of experimentalists and theorists alike.

In one of these experiments [8], a thermal Hall effect is observed in crystalline samples of $\mathrm{RuCl}_{3}$, a Mott insulator, in

Published by the American Physical Society under the terms of the Creative Commons Attribution 4.0 International license. Further distribution of this work must maintain attribution to the author(s) and the published article's title, journal citation, and DOI. which the charge degrees of freedom are frozen out ${ }^{1}$ and the heat transport [14] must be facilitated through charge-neutral modes. With the thermal conductance being quantized at a half-integer value, this points to the striking possibility of a Majorana fermion edge current forming in these systems. On the theoretical side, this is rationalized by the designation of $\mathrm{RuCl}_{3}$ as a Kitaev material [15], a special type of spin-orbitassisted Mott insulator [16,17], in which local spin-orbitentangled $j=1 / 2$ moments [18-20] form that are subject to

\footnotetext{
${ }^{1}$ The charge gap for $\mathrm{RuCl}_{3}$ has been reported to be within 0.22 to $1.2 \mathrm{eV}$ in experimental studies [10-13].

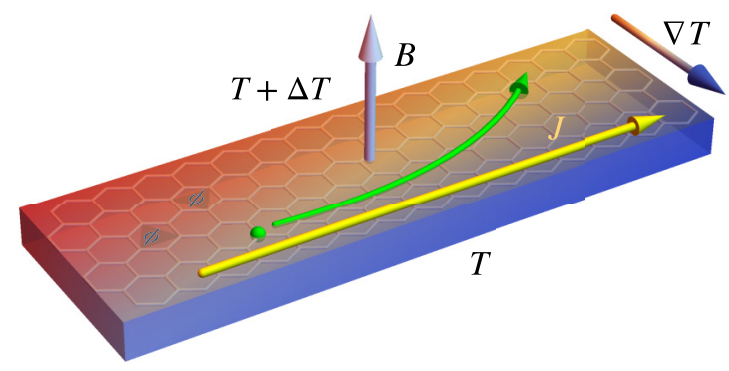

FIG. 1. Schematic illustration of the thermal Hall effect of charge-neutral spinons arising for a field-induced U(1) spin liquid with a spinon Fermi surface in honeycomb Kitaev materials in an external magnetic field.
} 
bond-directional exchanges [21] familiar from the celebrated Kitaev model [22]. The appeal of making such a direct connection to this elementary spin model comes from the fact that the latter exhibits a number of quantum spin liquid ground states [23,24]. Of these, the field-induced gapped topological spin liquid, often simply referred to as a Kitaev spin liquid, is a chiral spin liquid with gapless Majorana edge modes. As such it appears to be a natural fit to explain the quantized thermal Hall effect in $\mathrm{RuCl}_{3}$, in particular after considering the subtle interplay of gapless Majorana and phonon modes in such a chiral spin liquid $[25,26]$.

The observation of a finite but nonquantized thermal Hall effect is an even more general though still unusual phenomenon, which has been reported for not only a broad temperature and magnetic field range for $\mathrm{RuCl}_{3}$ [27,28] (in addition to the quantized regime), but also a number of other spin liquid candidate materials such as the kagome magnets volborthite [29] $\mathrm{Cu}_{3} \mathrm{~V}_{2} \mathrm{O}_{7}(\mathrm{OH})_{2} \cdot 2 \mathrm{H}_{2} \mathrm{O}$ and $\mathrm{Ca}$ kapellasite [30] $\mathrm{CaCu}_{3}(\mathrm{OH})_{6} \mathrm{Cl}_{2} \cdot 0.6 \mathrm{H}_{2} \mathrm{O}$, as well as the pyrochlore spin ice material [31] $\mathrm{Tb}_{2} \mathrm{Ti}_{2} \mathrm{O}_{7}$. This points to an alternative microscopic origin of charge-neutral thermal transport beyond the one sketched above for the gapped chiral spin liquid, which always leads to a quantized Hall effect $[25,26]$. Indeed, as some of us have recently pointed out in the context of kagome spin liquids [32], there is the possibility that a gapless quantum spin liquid can also exhibit a finite thermal Hall conductivity [33], even in the strong Mott insulating regime. The microscopic mechanism at play involves an interplay between the emergent charge-neutral spinon degrees of freedom and certain types of Dzyaloshinskii-Moriya interactions that lead to a twist in the spinon motion, thereby allowing for a transverse heat flow.

The purpose of this paper is to generalize this idea of emergent spinons mediating a charge-neutral transverse thermal Hall current to honeycomb Kitaev materials. The reason to do so is motivated by not only the experimental observations [27,28] for $\mathrm{RuCl}_{3}$ discussed above, but also recent reports of the emergence of a field-driven gapless U(1) spin liquid [34-36] in antiferromagnetic [37] Kitaev magnets paired with $a b$ initio modeling for the honeycomb Kitaev materials $\mathrm{RuCl}_{3}, \mathrm{Na}_{2} \mathrm{IrO}_{3}$, and $\alpha-\mathrm{Li}_{2} \mathrm{IrO}_{3}$, which report, in unison, a variety of additional interaction terms beyond a dominant bond-directional Kitaev exchange [17,38,39]. We demonstrate that in the presence of a general symmetry-allowed nextnearest neighbor Dzyaloshinskii-Moriya interaction the emergent gapless spinon degrees of freedom of such a field-driven $\mathrm{U}(1)$ spin liquid will indeed contribute to a transverse heat flow. This is not obvious a priori, since the net internal flux in every unit cell vanishes and there are thus no obvious spinon Landau levels or quantum oscillations. However, we argue along the lines of Ref. [32] that the spinons hopping between the second-neighbor sites will still experience an induced internal gauge flux. We explicitly demonstrate that this mechanism generates a nontrivial Berry curvature and thereby allows for a significant spinon thermal Hall effect, as schematically illustrated in Fig. 1. In reverse, this leads us to conclude that the observation of a finite nonquantized thermal Hall conductance in honeycomb Kitaev materials would point towards the possibility of non-Kitaev spin liquid regimes in these materials. We further substantiate this reasoning by considering a gapless Dirac spin liquid, a third possible spin liquid scenario besides the chiral spin liquid and the gapless spinon Fermi surface U(1) spin liquid, for which we arrive at a similar conclusion.

The discussion in the remainder of this paper is structured as follows. We begin in Sec. II with a detailed review of the symmetry-allowed microscopic spin model for the honeycomb iridates. In Sec. III we consider the U(1) spin liquid state with a neutral spinon Fermi surface and numerically estimate the spinon thermal Hall conductivity within linear-response theory. In Sec. IV we inspect an alternate scenario of a Dirac spin liquid. We conclude in Sec. $\mathrm{V}$ with a discussion of the results and the relevance of thermal transport measurements in the honeycomb Kitaev materials, including $\alpha-\mathrm{RuCl}_{3}$ and other Kitaev materials such as $\mathrm{H}_{3} \mathrm{LiIr}_{2} \mathrm{O}_{6}$. Technical details of our calculations and some further aspects of the materials are presented in the Appendixes.

\section{SPIN-ORBIT COUPLING AND SPIN MODEL}

To set the stage for our discussion, we start by providing a comprehensive and self-contained review of the microscopic physics of honeycomb Kitaev iridates. We focus particularly on the symmetry-allowed exchange interactions beyond a bare bond-directional Kitaev coupling and discuss the explicit form of the spin-orbit-induced Dzyaloshinskii-Moriya interaction. More extensive introductions to this basic microscopic physics can be found in early theory works on the iridates $[18,21,40]$ as well as more recent review articles $[15,41]$.

\section{A. Spin-orbit coupling and derivation of superexchange interaction}

The iridium atom has an atomic number $Z=77$ and thus the $5 d$ electrons of the iridium experience a much stronger spin-orbit coupling than the $3 d$ electrons of transition-metal ions. The full Hamiltonian of the iridates is given as [19]

$$
H=H_{\text {Ir-O }}+H_{\text {Ir-Ir }}+H_{\text {SOC }}+H_{\text {split }}+H_{\text {Ir-corr }}+H_{\text {O-corr }},
$$

where $H_{\text {Ir-O }}$ describes the hopping between the Ir $5 d\left(t_{2 g}\right)$ orbitals and the $\mathrm{O} p$ orbitals, $H_{\text {Ir-Ir }}$ is the direct hopping between the Ir $t_{2 g}$ electrons, $H_{\mathrm{SOC}}$ is the atomic spin-orbit coupling (SOC) of the Ir $t_{2 g}$ electrons, $H_{\text {split }}$ describes the crystal field splitting within $t_{2 g}$ orbitals, $H_{\text {Ir-corr }}$ is the electron correlation for the Ir $t_{2 g}$ electrons and is parametrized by various Kanamori interactions, and $H_{\mathrm{O}-\mathrm{corr}}$ is the electron correlation within the $\mathrm{O} p$ orbitals. In this extended Hubbard model, we have already made the approximation to truncate the upper $e_{g}$ orbitals. The $e_{g}-t_{2 g}$ splitting is of the order of $2 \mathrm{eV}$, and ignoring $e_{g}$ orbitals is a good approximation for the $5 d$ materials. This extended Hubbard model describes almost all of the iridates of interest. The Ir-O-Ir complex that often occurs in various iridates is given in Fig. 2.

This extended Hubbard model is similar to the three-band model for cuprates except that here multiple $t_{2 g}$ orbitals are involved and a strong spin-orbit coupling is present. In the context of cuprates, where the concept of the charge-transfer insulator is relevant, the further reduction of the three-band model to the single-band ( $t-J$ type) model is made possible through the observation of the Zhang-Rice singlets and the 
(a)

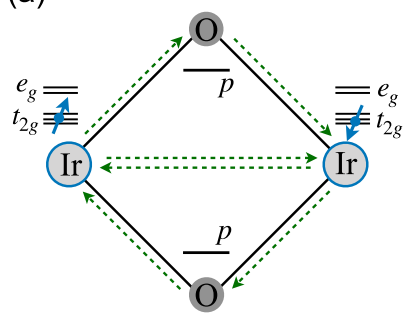

(b)

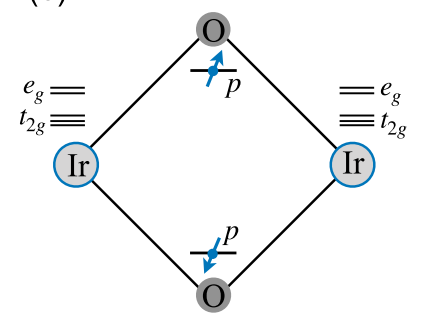

FIG. 2. Microscopics of honeycomb Kitaev materials. (a) Superexchange paths for different approximation schemes. (b) Intermediate electron-hole configuration for the superexchange through the oxygen atoms.

virtual sharing of the Zhang-Rice singlets between neighboring $\mathrm{Cu}-\mathrm{O}$ complexes.

Since we are interested in the Mott insulating regime, all the electrons are localized on the ionic sites and form a local moment. In the single-ion limit, there are five $5 d$ electrons on the $t_{2 g}$ shell and SOC is active at the linear order. In the single-electron basis, the atomic SOC appears as

$$
\begin{aligned}
H_{\mathrm{SOC}} & =\sum_{i} \lambda \boldsymbol{L}_{i} \cdot \boldsymbol{S}_{i} \\
& \Rightarrow-\sum_{i} \lambda \boldsymbol{l}_{i} \cdot \boldsymbol{S}_{i},
\end{aligned}
$$

where $\boldsymbol{L}_{i}$ operates on the five $5 d$ orbitals (including both $e_{g}$ and $t_{2 g}$ ) with $L=2, l$ operates within the lower $t_{2 g}$ triplets, and more importantly $l=1$. Thus, the SOC favors a lower quadruplet with $j=3 / 2$ and a upper doublet with $j=1 / 2$. Four electrons would completely fill the lower quadruplets, and the remaining electron occupies the upper $j=1 / 2$ (Kramers) doublet and gives rise to the effective spin-1/2 local moment. In the absence of the further splitting of the $t_{2 g}$ shell, the wave functions of the $j=1 / 2$ states are given as

$$
\begin{aligned}
& \left|j^{z}=\uparrow\right\rangle=\frac{1}{\sqrt{3}}[-i|x z, \downarrow\rangle+|y z, \downarrow\rangle+|x y, \uparrow\rangle], \\
& \left|j^{z}=\downarrow\right\rangle=\frac{1}{\sqrt{3}}[+i|x z, \uparrow\rangle+|y z, \uparrow\rangle-|x y, \downarrow\rangle] .
\end{aligned}
$$

Remarkably, because of the involvement of the orbitals, the Landé factor is $g=-2$ instead of the usual $g=2$ for the spin-only local moments. However, this sign difference cannot be measured in the magnetic susceptibility in which $g$ enters at an even power. In any real material, there exist small splittings among the $t_{2 g}$ orbitals. Such splittings lead to further modifications of the effective $j=1 / 2$ wave function and change the $g$ factor continuously from -2 to +2 for the limit that orbital degeneracy is completely broken and SOC is quenched. So the combination of SOC and $t_{2 g}$ splitting could generate a rather small magnetic moment. This can probably account for the small magnetic moments [42] of $\mathrm{Na}_{2} \mathrm{IrO}_{3}$ and $\mathrm{Li}_{2} \mathrm{IrO}_{3}$.

As the microscopic Hamiltonian in Eq. (1) contains many different interactions and couplings, there exist various approximation schemes to deal with this extended Hubbard model. One approximation is to keep the direct Ir-Ir hopping, the on-site SOC, and the Hubbard- $U$ correlation, i.e.,

$$
H_{A_{1}}=H_{\text {Ir-Ir }}+H_{\text {SOC }}+H_{\text {Ir-corr }} \text {. }
$$

One then projects the model onto the $j=1 / 2$ manifold. Remarkably, one obtains an apparent isotropy even though the effective spin itself contains a substantial orbital component, and the resulting spin model is a Heisenberg model [19,21]. Another approximation is to consider the superexchange through the oxygen with the starting Hamiltonian as

$$
H_{A_{2}}=H_{\text {Ir-O }}+H_{\text {SOC }}+H_{\text {Ir-corr }}+H_{\text {O-corr }} \text {. }
$$

In leading order, the Heisenberg term just vanishes completely. References [19,21] further considered the splitting among the $t_{2 g}$ orbitals and obtained a highly anisotropic spin model. For the type- $x$ bond that are in the $y z$ plane, the superexchange interaction was found to be [19]

$$
H_{\mathrm{ex}_{1}}=\sum_{\langle i j\rangle \in x} J\left[-S_{i}^{x} S_{j}^{x}+S_{i}^{y} S_{j}^{y}+S_{i}^{z} S_{j}^{z}\right]
$$

and the exchange interaction of other bonds is obtained by simple permutations. Jackeli and Khalliulin further considered the effect of the Hunds coupling in Ref. [21] and remarkably obtained a pure Kitaev interaction for the honeycomb iridate with

$$
H_{\mathrm{ex}_{2}}=\sum_{\langle i j\rangle \in x}-K S_{i}^{x} S_{j}^{x}
$$

on the type- $x$ bond, and the interactions on other bonds are obtained by simple permutations. The anisotropic nature of the superexchange interactions arises from the spin-orbitentangled nature and the significant orbital content of the $\mathrm{Ir}^{4+}$ local moments.

We close by noting that due to the complication of the extended Hubbard model, further theoretical refinements, such as the application of a Schrieffer-Wolff transformation, could lead to more complete predictions for the exchange interaction. Another noteworthy observation concerns the importance of the intermediate electron configuration on the interstitial oxygen ions. One could have two holes on the same oxygen atom, or one hole on one oxygen atom and the other hole on the other oxygen atom [see Fig. 2(b)]. If one considers these intermediate configurations on oxygen atoms, the approximation of integrating out the oxygen $2 p$ orbitals or states may not be the best approximation, especially when electron correlations on the $2 p$ orbitals are included.

\section{B. Antisymmetric Dzyaloshinskii-Moriya interaction}

For $3 d$ transition-metal compounds with weak SOC, antisymmetric Dzyaloshinskii-Moriya interactions $[43,44]$ are expected as a higher-order perturbation rather than the pure Heisenberg one when the magnetic bonds have no inversion center. For magnets with spin-orbit-entangled local moments, the original perturbative treatment of SOC [44] to obtain the Dzyaloshinskii-Moriya interactions no longer applies, but one can rely on a symmetry analysis and Moriya's rules to constrain the Dzyaloshinskii-Moriya interactions. For the twodimensional honeycomb lattice, the first-neighbor magnetic sites have inversion symmetry, and thus the first-neighbor Dzyaloshinskii-Moriya interaction is prohibited. However, a second-neighbor Dzyaloshinskii-Moriya interaction is allowed by symmetry since the second-neighbor magnetic bonds have no inversion center. According to Moriya's rules 
(a)

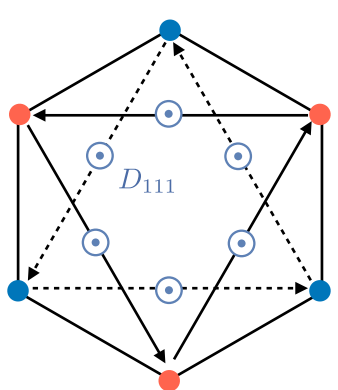

(b)

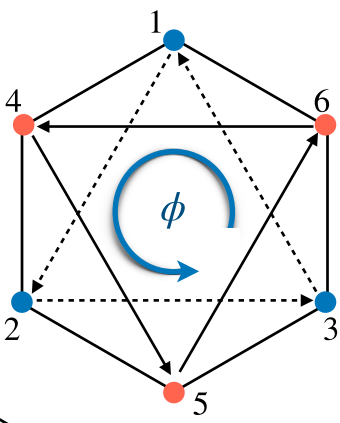

(c)

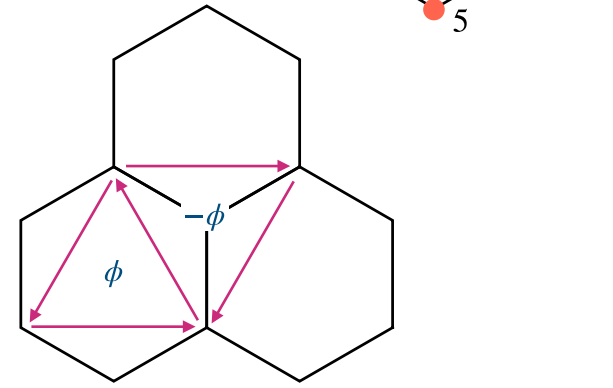

FIG. 3. Dzyaloshinskii-Moriya interactions. (a) Symmetry allowed Dzyaloshinskii-Moriya interactions between second neighbors on the honeycomb lattice, where $D_{111}$ is the [111] component. The arrows specify the order of the cross product $S_{i} \times S_{j}$. The two sublattices are labeled by colors. (b) Schematic view of the gauge flux $\phi$ induced by the external magnetic field in the presence of the next-nearest-neighbor Dzyaloshinskii-Moriya interaction. (c) The net flux in one unit cell is zero and the space translation symmetry is well preserved.

[44], there are components of $\boldsymbol{D}_{i j}$ perpendicular to the planes with strength $D_{111}$, as schematically depicted in Fig. 3(a), and all the in-plane components vanish when the honeycomb plane is a mirror plane of the crystal structure. Therefore, a representative Dzyaloshinskii-Moriya interaction of the honeycomb lattice Mott insulator up to the second neighbor has the form

$$
H_{\mathrm{DM}}=\sum_{\langle\langle i, j\rangle\rangle} \boldsymbol{D}_{i j} \cdot \boldsymbol{S}_{i} \times \boldsymbol{S}_{j}
$$

For example, it has been estimated [38] that a large second-neighbor Dzyaloshinskii-Moriya term $\left|\boldsymbol{D}_{i j}\right|>4 \mathrm{meV}$ is present for the Kitaev material $\alpha-\mathrm{Li}_{2} \mathrm{IrO}_{3}$, which, however, is often not considered in the literature.

With these microscopic considerations in place, we note again that our purpose in the following is not to solve for the ground state of a specific Hamiltonian. Instead, we assume that the system stabilizes, in the presence of an external magnetic field, a non-Kitaev spin liquid, as suggested by numerical studies [34-36,45], and clarify how the elementary spinons in these spin liquids acquire an emergent Lorentz force in the external field through the Dzyaloshinskii-Moriya interaction. Due to the Zeeman coupling, a moderate magnetic field partially polarizes the spins and generates a finite second-neighbor scalar spin chirality on the lattice through the Dzyaloshinskii-Moriya interaction. This in turn induces an internal gauge flux for the spinons, as we will show in the following, and ultimately gives rise to a thermal Hall effect.

\section{THERMAL HALL EFFECT FOR SPIN LIQUID WITH A SPINON FERMI SURFACE}

As the first example of a non-Kitaev spin liquid we consider the scenario of a $U(1)$ spin liquid with a spinon Fermi surface. This is motivated by a recent string of numerical works [34-36] that report strong evidence for the emergence of such a U(1) spin liquid as an intermediate gapless phase in the antiferromagnetic Kitaev model before entering the high-field trivial polarized state.

In more technical terms, the $\mathrm{U}(1)$ spin liquid describes a highly entangled quantum state with gapless fermionic spinons coupled to a massless $\mathrm{U}(1)$ gauge field. On a meanfield level, a Hamiltonian for the neutral spinon Fermi surface state can be constructed as

$$
H_{\mathrm{MF}}=H_{\mathrm{hop}}+H_{B},
$$

where $H_{\text {hop }}$ contains only spinon hopping operators on the honeycomb lattice and

$$
H_{B}=-\frac{B}{2} \sum_{i, \alpha \beta} f_{i, \alpha}^{\dagger}\left(\sigma_{x}+\sigma_{y}+\sigma_{z}\right)_{\alpha \beta} f_{i, \beta}
$$

represents the Zeeman coupling to an external magnetic field $B$ along the [111] direction, with $f_{i, \alpha}\left(f_{i, \alpha}^{\dagger}\right)$ the spinon annihilation (creation) operator at site $i$. The [111] direction is normal to the honeycomb plane. By studying the relation between the relevant projective symmetry groups [46], three kinds of U(1) spin liquids are obtained [35] that are connected to the Kitaev $\mathbb{Z}_{2}$ spin liquid state through a continuous phase transition without symmetry breaking. Moreover, only one of them, labeled $U_{1} A_{k=0}$ in Ref. [35], was shown to support robust spinon Fermi surfaces. A representative mean-field Hamiltonian for such a state, i.e., a U(1) spin liquid with a neutral spinon Fermi surface on the honeycomb lattice, is given in Appendix A. We will use this mean-field Hamiltonian as our starting point in the following discussion.

\section{A. Field induced internal flux via Dzyaloshinskii-Moriya interaction}

For the U(1) spin liquid with a spinon Fermi surface in the weak Mott regime, by switching on an external magnetic field, a ring exchange interaction derived from the Hubbard model can contribute to the thermal Hall conductivity $[33,47,48]$. It was originally proposed for the well-known triangular lattice organic spin liquid candidate $\kappa-(\mathrm{ET})_{2} \mathrm{Cu}_{2}(\mathrm{CN})_{3}$, due to its proximity to the Mott transition [48]. However, since we are working in the strong Mott regime, such a mechanism does not apply because of the large charge gap. On the other hand, as we have mentioned in Sec. II B, the combination of the microscopic Dzyaloshinskii-Moriya interaction and Zeeman coupling further induces an internal U(1) gauge flux distribution on the honeycomb plane.

More explicitly, in the U(1) spin liquid phase, gauge fluctuations are described by a continuous lattice $\mathrm{U}(1)$ gauge theory and the internal gauge flux is related to the underlying spin chirality as [49-51]

$$
\sin \phi=\frac{1}{2} \boldsymbol{S}_{1} \cdot \boldsymbol{S}_{2} \times \boldsymbol{S}_{3},
$$


where $\phi$ is the flux defined on the triangular plaquette formed by three second-neighbor sites of the honeycomb lattice. Following previous work by some of us [32], one can then establish

$$
\sin \phi \simeq \lambda D_{111} \chi B / 2
$$

under an external magnetic field $B$ (with $\chi$ the magnetic susceptibility). Considering an elementary hexagon as schematically illustrated in Fig. 3(b), the flux through the triangle formed by sites 1,2 , and 3 in the counterclockwise direction corresponds to $\phi$. Similarly, the flux through the triangle formed by sites 4,5 , and 6 in the counterclockwise direction is still $\phi$, i.e., the fluxes of the triangles formed by the second-neighbor bonds in one hexagon are exactly equal for two sublattices. However, the flux for the triangle formed by the second neighbor bonds of three different hexagons acquires a minus sign if adopting the counterclockwise loop convention. That is to say, the net flux in one unit cell is zero and the space translation symmetry is not destroyed, as shown in Fig. 3(c), where we plot only the triangles formed by one sublattice for simplicity (with an analogous situation for the other sublattice). The spinons carry emergent U(1) gauge charges and are minimally coupled to the U(1) gauge field; thus the spinons will feel such gauge flux as the spinons hop between second-neighbor sites on the lattice. It is necessary to stress that the first-neighbor spinon hopping does not pick up any phase since the net flux in a unit cell is zero, much like the Haldane model for spinless fermions.

\section{B. Reconstructed fermionic spinon bands}

Physically, as the spinon moves on the lattice, it will experience a Lorentz force from the induced internal flux. On a semiclassical level, the spinon motion will be twisted and reflected, resulting in a spinon thermal Hall effect. On a quantum mechanical level, this effect can be understood from the spinon Berry curvature, which we explain below.

The internal gauge flux pattern is depicted in Figs. 3(b) and 3(c). To capture the flux, we modify the spinon meanfield Hamiltonian by adding the $U(1)$ gauge potential to the next-nearest-neighbor hopping terms. This immediately leads to a modified spinon dispersion. Combining the two sublattices with the two spin labels, a total of four spinon bands are obtained, which are half filled. As depicted in Fig. 4(a), the internal U(1) gauge flux reconstructs the spinon bands and there still exist Fermi pockets. When the magnetic field exceeds some critical value where the pockets vanish, according to Polyakov's argument [52], the dynamical U(1) gauge field will be confined due to nonperturbative instanton events and the system enters a trivial polarized state. To describe the thermal Hall effect, we therefore focus on the stable, deconfined spin liquid regime and further clarify the induced internal gauge flux that would contribute to the spinon thermal Hall effect.

Let us now explicitly demonstrate the finite thermal Hall conductivity for the spin liquid in the presence of magnetic field. With the aid of Luttinger's pseudogravitational potential [53], the thermal Hall conductivity formula for a general noninteracting fermionic system with a nonzero chemical (a)

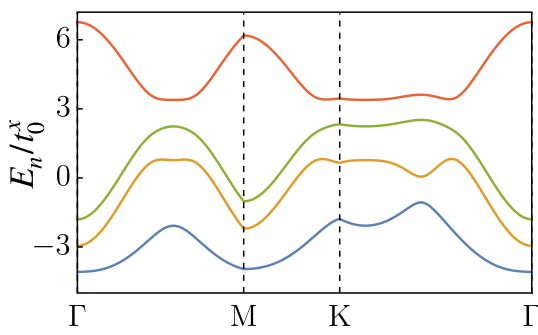

(b)

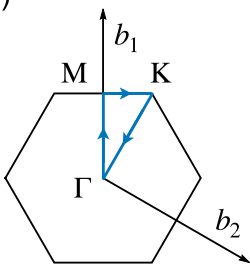

FIG. 4. (a) Representative spinon dispersions for the nonzero mean-field parameters $\left(s_{3}, t_{0}^{x}, t_{0}^{y}\right)=(-1,-1,-0.2), \quad\left(\tilde{s}_{0}, \tilde{s}_{3}\right)=$ $(-0.2,0.2)$, and $\left(\tilde{t}_{0}^{x}, \tilde{t}_{0}^{z}, \tilde{t}_{3}^{x}, \tilde{t}_{3}^{z}\right)=(-0.2,-0.2,-0.02,-0.02)$ along the high-symmetry line. Here the magnetic field is set as $B=1$ and the induced gauge flux $\phi=\pi / 20$. (b) Brillouin zone of the honeycomb lattice with reciprocal lattice vectors $\boldsymbol{b}_{1}=2 \pi(0,2 / \sqrt{3})$ and $\boldsymbol{b}_{2}=2 \pi(1,-1 / \sqrt{3})$. The arrows indicate the direction of the high-symmetry line in (a).

potential $\mu$ can be obtained [54] as

$$
\kappa_{x y}=-\frac{1}{T} \int d \epsilon(\epsilon-\mu)^{2} \frac{\partial f(\epsilon, \mu, T)}{\partial \epsilon} \sigma_{x y}(\epsilon) .
$$

Here $f(\epsilon, \mu, T)=1 /\left[e^{\beta(\epsilon-\mu)}+1\right]$ is the Fermi-Dirac distribution and the derivative of the distribution function $\partial f(\epsilon, \mu, T) / \partial \epsilon$ indicates that the integral dominates around the Fermi energy. Moreover,

$$
\sigma_{x y}(\epsilon)=-\frac{1}{\hbar} \sum_{k, \xi_{n, k}<\epsilon} \Omega_{n, k}
$$

is the zero-temperature anomalous Hall coefficient for a system with the chemical potential $\epsilon$. Here $\Omega_{n \boldsymbol{k}}$ is the Berry curvature for the fermions, which is defined as

$$
\Omega_{n \boldsymbol{k}}=-2 \operatorname{Im}\left\langle\frac{\partial u_{n \boldsymbol{k}}}{\partial k_{x}} \mid \frac{\partial u_{n \boldsymbol{k}}}{\partial k_{y}}\right\rangle,
$$

with eigenstate $\left|u_{n k}\right\rangle$ for bands indexed by $n$. Equation (15) suggests that the thermal Hall conductivity is directly related to the spinon Berry curvature in momentum space and a finite Berry curvature is necessarily required to generate $\kappa_{x y}$. We show below that the magnetic-field-induced internal U(1) gauge flux does indeed generate a finite Berry curvature and we can use Eq. (15) as our basis to calculate the thermal Hall conductivity for the spinon metal in a U(1) spin liquid. As depicted in Fig. 5(a), one can see that the modified mean-field Hamiltonian generates nontrivial spinon Berry curvatures for each band due to the influence of the induced internal gauge flux. The numerical results for the thermal Hall conductivity are presented in Fig. 5(b). For a second-neighbor hopping coefficient $t_{2}=0.5 t_{1}$, we obtain a monotonic temperature dependence of $\kappa_{x y} /\left(k_{B}^{2} T / \hbar\right)$. In the zero-temperature limit, it trends to a nonzero and nonquantized value. In the finitetemperature region, the thermal Hall conductivity decreases monotonically and finally vanishes at high temperatures. The vanishing thermal Hall conductivity in the high-temperature region originates from the almost equally populated spinon bands and the corresponding Berry curvature cancellation. 
(a)

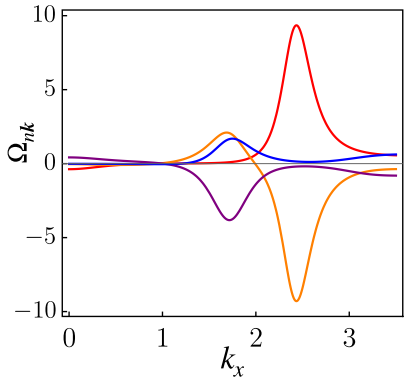

(b)

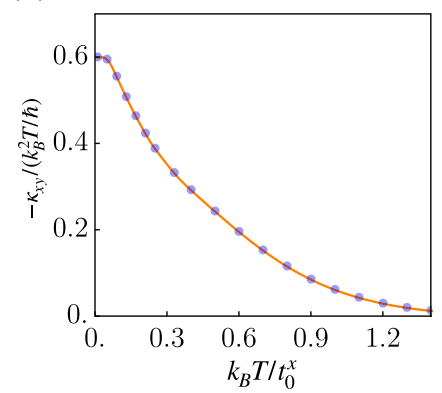

FIG. 5. (a) Berry curvature for different energy bands with all the parameters set as in Fig. 4. The red, orange, purple, and blue lines are for the first (lowest), second, third, and fourth spinon bands, respectively. (b) Corresponding evaluation of the thermal Hall conductivity as a function of temperature.

\section{Stability of the U(1) spin liquid}

Numerical evidence for a U(1) spin liquid in the Kitaev honeycomb model was recently reported for an intermediatemagnetic-field range [34-36]. Since the Kitaev spin liquid can be understood as the $p$-wave pairing of the Abrikosov fermionic spinons, if one replaces the Majorana fermion representation with the fermionic spinon representation [55], this intermediate field $U(1)$ spin liquid with spinon Fermi surface can be understood as the Pauling limit of the $p$-wave pairing where the pairing is removed. Here we investigate the stability of this U(1) spin liquid to a finite Dzyaloshinskii-Moriya interaction using exact diagonalization techniques. For fields close to the (111) direction the intermediate $\mathrm{U}(1)$ spin liquid occurs in a field range of $h \sim 0.35 K-0.60 K$ (where $h$ is the field magnitude, $h=|\boldsymbol{h}|$ ). We focus on this field range and consider the effects of adding a Dzyaloshinskii-Moriya interaction of the form given in Eq. (10). The Hamiltonian is thus

$$
H=\sum_{\langle i j\rangle \in \gamma} K S_{i}^{\gamma} S_{j}^{\gamma}+\sum_{\langle i, j\rangle} \boldsymbol{D}_{i j} \cdot \boldsymbol{S}_{i} \times \boldsymbol{S}_{j}-\sum_{i} \boldsymbol{h}_{i} \cdot \boldsymbol{S}_{i} .
$$

In Fig. 6 we show the resulting phase diagram, with the phase boundaries determined using a combination of the second derivative of the ground-state energy and the ground-state fidelity [56]. The U(1) spin liquid region is stable up to a maximal Dzyaloshinskii-Moriya interaction of about $|\boldsymbol{D}| \sim 0.025 \mathrm{~K}$. We should note, however, that additional interactions, relevant for real Kitaev materials, could further increase or decrease the stability of the $\mathrm{U}(1)$ spin liquid against the effects of the finite Dzyaloshinskii-Moriya term. In any case, the $\mathrm{U}(1)$ spin liquid is stable to adding finite, though small, Dzyaloshinskii-Moriya interactions. This justifies our starting point of $U(1)$ spin liquid even in the presence of Dzyaloshinskii-Moriya interactions.

\section{THERMAL HALL EFFECT FOR DIRAC SPIN LIQUID}

For particular magnetic-field directions on the honeycomb plane, a gapless Dirac spin liquid and a gapped KalmeyerLaughlin-type [57] chiral spin liquid were both numerically obtained in Ref. [45] for certain parameter regimes of the socalled Kitaev- $\Gamma$ model, a microscopic model with additional

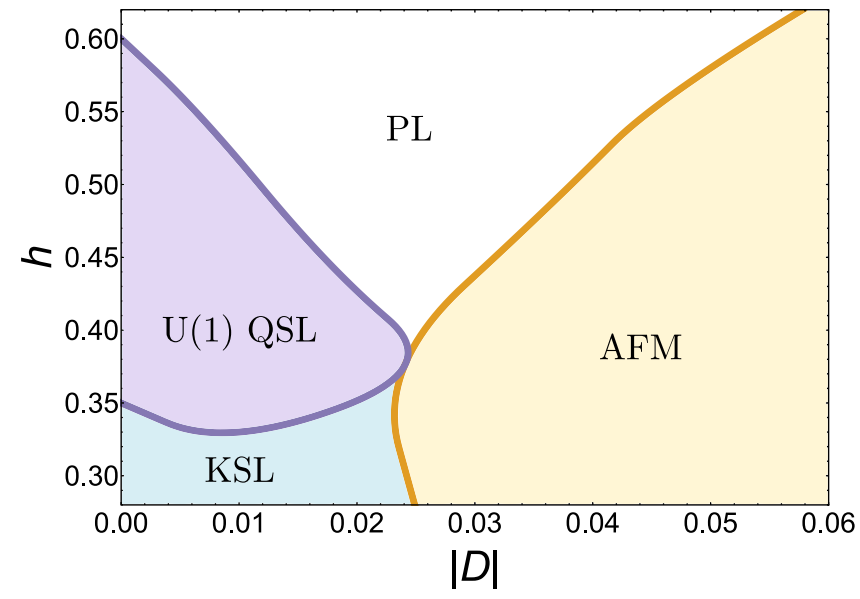

FIG. 6. Phase diagram for an extended Kitaev model in the combined presence of a finite Dzyaloshinskii-Moriya interaction, of strength $|\boldsymbol{D}|$, and a finite magnetic field, of magnitude $h$ and oriented close to the [111] direction. The energy unit is in the Kitaev coupling $K$ of Eq. (18). Here U(1) QSL specifically refers to our spinon Fermi surface U(1) spin liquid, KSL refers to the Kitaev spin liquid, AFM refers to the antiferromagnetic ordered state, and PL refers to the polarized state.

symmetric off-diagonal $\Gamma$ terms beyond the Kitaev exchange that has been argued $[17,38,39]$ to be particularly relevant to experimental Kitaev materials.

The gapped chiral spin liquid can be characterized by the net Chern number of the occupied spinon bands. In addition, note that the ansatz of such a chiral spin liquid readily breaks both time-reversal symmetry $\mathcal{T}$ and reflection $P$, while their combination $P \mathcal{T}$ is well preserved. Generically, this leads to a nonvanishing expectation value for the chiral order parameter $\boldsymbol{S}_{i} \cdot\left(\boldsymbol{S}_{j} \times \boldsymbol{S}_{k}\right)$, where $i, j$, and $k$ are three nearby sites. The chiral spin liquid is effectively described by the Chern-Simons theory with semion topological order; in particular, this state has chiral edge modes and would show an integer-quantized thermal Hall effect. Thus we are not going to further discuss the influence of the induced internal gauge flux on this state due to the Chern-Simons term in the theory for gauge fluctuations.

Here we consider the situation where the system stabilizes and stays in a gapless Dirac spin liquid state. Such a Dirac spin liquid is a deconfined state with Dirac band touchings at the Fermi level and its low-energy effective theory is described by the Dirac equation. Usually, a Dirac spin liquid has no thermal Hall effect associated with it. A representative spinon dispersion for the Dirac spin liquid realized in the Kitaev- $\Gamma$ model for the honeycomb lattice is depicted in Fig. 7(a), where we have adopted the spinon mean-field Hamiltonian constructed in Ref. [45] (see Appendix B for details). One can see that, at the Fermi level, there is a Dirac band touching at the $K$ point of the Brillouin zone. We assume that this deconfined spin liquid state is stabilized in a finite region of the phase diagram and the presence of the second-neighbor Dzyaloshinskii-Moriya interaction would not destroy it.

As in the spinon Fermi surface U(1) spin liquid case, the gauge fluctuations of the Dirac spin liquid are described by a U(1) gauge theory, thus the external magnetic 

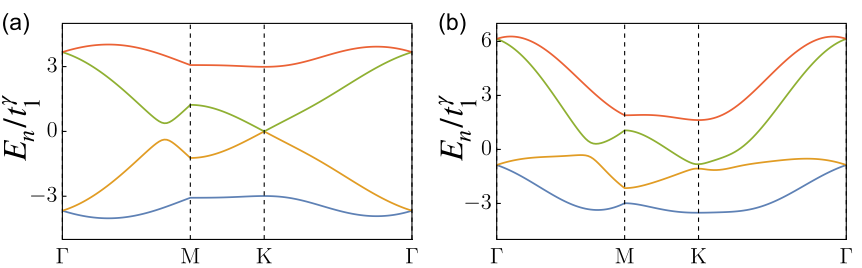

FIG. 7. (a) Spinon dispersion for the Dirac spin liquid. (b) Induced flux for the second-neighbor hopping terms reconstructs the spinon bands and the resulting state is a spinon Fermi surface spin liquid. There is a Fermi pocket around the $K$ point of the Brillouin zone.

field also induces an internal gauge flux for the secondneighbor spinon hopping channels through the secondneighbor Dzyaloshinskii-Moriya interaction and leads to a spinon thermal Hall effect. Such flux will reconstruct the spinon bands and the resulting state is a spinon Fermi surface spin liquid with a Fermi pocket around the $K$ point, as shown in Fig. 7(b). Although the Dirac band touching is eliminated, when we consider the influence of the gauge flux, the system is still in a deconfined phase since the matter field is also gapless and the gap between the second and third bands is not relevant. Following a procedure similar to that for the calculation in Sec. III, in Fig. 8 we plot the temperature dependence of the thermal Hall conductivity for this state. In contrast to Fig. 5(b), the ratio of thermal Hall conductivity and temperature for this state increases rapidly with temperature and then decreases gradually after reaching a maximum in a finite-temperature region. Such a different temperature dependence originates from the special spinon dispersion and the corresponding spinon Berry curvature of this state. The vanishing of the thermal Hall conductivity in the high-temperature region can again be explained by the Berry curvature cancellation of different spinon bands.

\section{DISCUSSION}

While the original motivation for the exploration of the growing family of Kitaev materials [15-17] might have been to discover an experimental realization of the Kitaev spin liquid [22], i.e., a non-Abelian chiral spin liquid with a

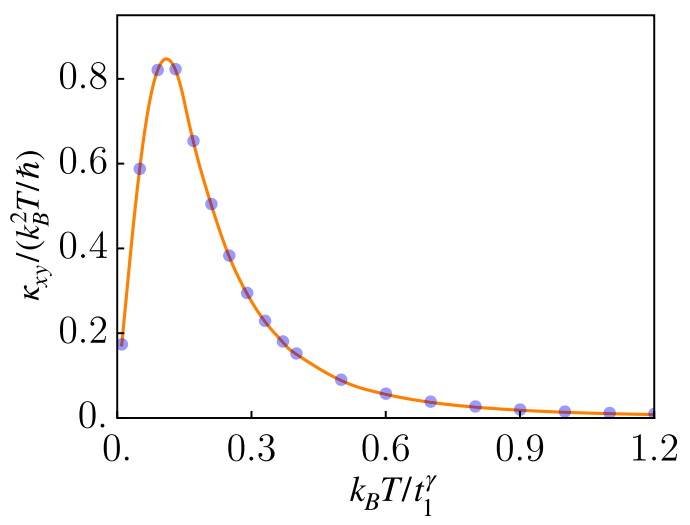

FIG. 8. Thermal Hall conductivity for Dirac spin liquid as a function of temperature at the gauge flux $\phi=\pi / 10$; here $t_{2}$ is set as $0.4 t_{1}^{\gamma}$. gapless Majorana edge current, it is becoming increasingly clear that these materials might also harbor other types of spin liquids [34-36,45,58]. Theoretical investigations suggest that this is particularly true when considering field-induced phases, for which the emergence of a U(1) spin liquid with a spinon Fermi surface [34-36], a Dirac spin liquid [45], and Abelian chiral spin liquids [45] have been proposed. In the absence of magnetic fields, additional types of $\mathbb{Z}_{2}$ spin liquids, beyond the ones known from the bare Kitaev model, have been proposed to arise from strong spin-orbit coupling [58]. However, narrowing in on a precise theoretical prediction starting from $a b$ initio modeling [17,38] for a given Kitaev material remains a formidable challenge, not least because of the myriad of additional couplings that are at play in these materials beyond the pure Kitaev exchange [39], including isotropic Heisenberg interactions of varying range and spin-orbit-induced off-diagonal spin exchanges or Dzyaloshinskii-Moriya interactions. This is particularly so for non-honeycomb-based iridates with a three-dimensional structure, as more complex lattice geometries allow for even more types of symmetry-allowed magnetic interactions.

It might thus be a better strategy to instead start from the potential spin liquid states and to single out experimental signatures that allow one to distinguish between these different nonmagnetic states. As we have argued in this paper, the observation of a thermal Hall effect is precisely such a measure. In the absence of magnetic orders, it allows one to single out the nature of the potential candidate spin liquids: a finite but nonquantized thermal Hall effect is indicative of a gapless spin liquid, with either a spinon Fermi surface or a Dirac spectrum, while a quantized thermal Hall effect exposes a gapped chiral spin liquid whose Abelian versus nonAbelian character is reflected in the integer versus half-integer quantization of the edge modes.

\section{Application to $\mathrm{H}_{3} \mathrm{LiIr}_{2} \mathrm{O}_{6}$}

Among the honeycomb Kitaev materials, the recently synthesized $\mathrm{H}_{3} \mathrm{LiIr}_{2} \mathrm{O}_{6}$ [59] and $\mathrm{Cu}_{2} \mathrm{IrO}_{3}$ [60] stand out as the only materials that remain disordered down to the lowest measured temperature. As such they might be the best candidate materials to date to exhibit a (gapless) Kitaev spin liquid even in the absence of magnetic field. We direct our discussion to $\mathrm{H}_{3} \mathrm{LiIr}_{2} \mathrm{O}_{6}$ specifically. Experimentally, this system exhibits a constant susceptibility and sublinear power-law heat capacity at low temperatures [59]. The observation of constant magnetic susceptibility might not be unexpected in light of the fact that the Ir $5 d$ electrons are subject to strong SOC, which in turn renders the notion that the magnetization remains a good quantum number down to zero temperature invalid as discussed early on [19]. To rationalize the unusual scaling behavior of the specific heat, which must be rooted in a divergent low-energy density of states, several explanations have been put forward that start from the gapless Kitaev spin liquid and consider the effect of additional perturbations, such as a residual interlayer coupling [61] or disorder effects [62-65]. Indeed, ab initio calculations indicate that the local $j=1 / 2$ moments experience a significant amount of quenched bond disorder arising from structural disorder of the $\mathrm{H}$ ions $[62,64]$. On a phenomenological level, the formation of a disorder-induced 
random singlet phase has been put forward [65]. ${ }^{2}$ Considering these different scenarios, one naturally arrives at the question of how one can distinguish the different potential origins of the apparent nonmagnetic behavior of $\mathrm{H}_{3} \mathrm{LiIr}_{2} \mathrm{O}_{6}$ (disorder effects versus the formation of a random singlet phase or the emergence of a spin liquid) in experimental probes.

As we have argued above, performing thermal Hall measurements for small external magnetic fields on the single-crystalline samples of $\mathrm{H}_{3} \mathrm{LiIr}_{2} \mathrm{O}_{6}$, which have recently become available, would provide distinct insight, with the observation of a thermal Hall signature being direct evidence of a spin liquid scenario. If the disorder is in fact weak and the system is indeed in a gapless Kitaev spin liquid, the field will induce a transition to a fully gapped Kitaev spin liquid that may overcome the disorder effect and show a half-quantized thermal Hall effect, similar to what has been observed for $\mathrm{RuCl}_{3}$. If one instead observes a finite but nonquantized thermal Hall effect this would count as evidence for the formation of a non-Kitaev spin liquid, with either a spinon Fermi surface or a Dirac cone spectrum.

\section{CONCLUSION}

With our present study we have completed an analysis of the thermal Hall signatures of various Kitaev and non-Kitaev spin liquids that have been discussed as candidate phases in the context of Kitaev materials in an external magnetic field. Complementing earlier studies on the conventional Kitaev spin liquid, a chiral spin liquid with a topological Majorana fermion band structure, we have considered in detail the spinon thermal Hall effect arising for various non-Kitaev spin liquids, in particular gapless U(1) spin liquids with a spinon Fermi surface [34-36], Dirac spin liquids, and variants of Abelian chiral spin liquids [45].

The mechanism for the appearance of a finite thermal Hall effect in the case of U(1) spin liquids, namely, the interplay of Dzyaloshinskii-Moriya interactions and Zeeman coupling, also results in a specific angular dependence of the sign of the measured thermal Hall conductivity. Specifically, the sign of the Hall conductivity is fixed by the sign of the dot product between the Dzyaloshinskii-Moriya vector with the external magnetic field, i.e., whether they are parallel or antiparallel. In

\footnotetext{
${ }^{2}$ The formation of such a random singlet phase has been conceptualized as an extension of a one-dimensional random singlet phase to high dimensions with spin-orbit anisotropy [65]. Note, however, that there are some key differences from the one-dimensional scenario. For a random antiferromagnetic spin- $1 / 2$ chain, the random singlet phase can be obtained in a rather elegant calculation performing a real-space renormalization group and master equation flow [66-68], which is asymptotically exact. However, this asymptotic exactness does not easily generalize as has been recognized for other onedimensional problems, such as disordered boson chains [69]. In higher spatial dimensions, the higher connectivity of every site and the sign of the interactions may further complicate the problem, and the formulation of a strong-disorder renormalization group approach for two-dimensional systems [70-72] remains a formidable challenge. As such, endowing the proposal for a two-dimensional random singlet phase with a more substantial theoretical footing may remain an open and interesting subject at this stage.
}

the case of $\alpha-\mathrm{RuCl}_{3}$, where the Dzyaloshinskii-Moriya vector is perpendicular to the honeycomb planes, this means that the sign is simply given by the sign of the out-of-plane component of the field, $\operatorname{sgn}\left(B_{111}\right)$.

It is important to note that a finite thermal Hall effect can also arise in a magnetically ordered state, where it arises from a nontrivial Berry curvature of the elementary magnon bands [73]. As such, it is of paramount importance to first establish whether a given material exhibits any ordering tendencies in the temperature and magnetic-field regime of interest, in order to distinguish whether a possible thermal Hall signature arises from conventional magnons or in fact spinons, which would be a strong indication of the fractionalization inherently connected to quantum spin liquid formation.

Besides the thermal Hall effect, there have been several recent theoretical works that attempt to understand the magnetic-field effect on the spinon Fermi surface state. For the case of strong Mott insulators, where only the Zeeman coupling needs to be considered, Ref. [74] established the spectral evolution of the spinon continuum and a spectral crossing in the continuum within the free spinon mean-field theory. More recently, Ref. [75] included the effect of spinongauge coupling as well as short-range spinon interactions to predict the existence of a new collective mode dubbed spinon wave mode. For the case of weak Mott insulators, recent work [76] considered the orbital coupling to the magnetic field [48] rather than a Zeeman coupling to estimate the cyclotron resonance of the spinon Fermi surface state.

\section{ACKNOWLEDGMENTS}

Y.H.G. and G.C. thank Jia-Wei Mei and Shiyan Li for an update on the experimental analysis of $\mathrm{RuCl}_{3}$ and Xuefeng Sun from USTC for clarifying the experimental setup. This work was supported by the Ministry of Science and Technology of China through Grants No.2016YFA0301001, No. 2016YFA0300500, and No. 2018YFGH000095, by the General Research Fund Grant No. 17303819 from the Research Grants Council of Hong Kong, and by the Deutsche Forschungsgemeinschaft (German Research Foundation), Projects No. 277101999 and No. 277146847, TRR 183 (Project B01) and CRC 1238 (Project C02).

\section{APPENDIX A: SPINON FERMI SURFACE MEAN-FIELD HAMILTONIAN}

When we discuss the spinon Fermi surface spin liquid realized in this system under an intermediate magnetic field, we mainly follow the analysis in Ref. [35] and use the results and conclusions therein. In the numerical calculation, the physical spin model that was used is the original Kitaev model with magnetic field and exchange anisotropy. For the convenience of the presentation in the work, we also list one of their mean-field Hamiltonians on which we focus here. In the momentum space, the spinon mean-field Hamiltonian for the $U_{1} A_{k=0}$ state with a neutral spinon Fermi surface has the form

$$
H_{\mathrm{NFS}}=\sum_{k} \Psi_{k}^{\dagger}\left(h_{0 k}+h_{1 k}+h_{2 k}\right) \Psi_{k},
$$


with the $\mathbf{k}$-space basis $\Psi_{\boldsymbol{k}}=\left(a_{\boldsymbol{k} \uparrow}, a_{\boldsymbol{k} \downarrow}, b_{\boldsymbol{k} \uparrow}, b_{\boldsymbol{k} \downarrow}\right)^{T}$, where $a_{\mathbf{k}}$ and $b_{\mathbf{k}}$ are for $A$ and $B$ sublattices, respectively. Moreover, the numbers 0,1 , and 2 denote on-site, nearest-neighbor, and next-nearest-neighbor terms, respectively, as defined below.

The on-site terms are given by

$$
h_{0}=-\mu \tau_{0} \sigma_{0}-\frac{B}{8} \tau_{0}\left(\sigma_{x}+\sigma_{y}+\sigma_{z}\right),
$$

where $\mu$ is the spinon chemical potential and would be selfconsistently calculated by the Hilbert space constraint

$$
\sum_{\sigma} f_{i \sigma}^{\dagger} f_{i \sigma}=1
$$

Nearest-neighbor terms are

$$
\begin{gathered}
h_{1 \boldsymbol{k}}=-\left(\begin{array}{cc}
0 & D_{\boldsymbol{k}} \\
D_{\boldsymbol{k}}^{\dagger} & 0
\end{array}\right) \\
D_{\boldsymbol{k}}=\left(s_{3} \sigma_{0}+t_{0}^{x} \sigma_{x}+t_{0}^{y} \sigma_{y}+t_{0}^{y} \sigma_{z}\right) e^{-i k_{1}} \\
+\left(s_{3} \sigma_{0}+t_{0}^{y} \sigma_{x}+t_{0}^{x} \sigma_{y}+t_{0}^{y} \sigma_{z}\right) e^{-i k_{2}} \\
+\left(s_{3} \sigma_{0}+t_{0}^{y} \sigma_{x}+t_{0}^{y} \sigma_{y}+t_{0}^{x} \sigma_{z}\right)
\end{gathered}
$$

and next-nearest-neighbor terms are

$$
\begin{gathered}
h_{2 k}=-\left(\begin{array}{cc}
A_{\boldsymbol{k}} & 0 \\
0 & B_{\boldsymbol{k}}
\end{array}\right) \\
A_{\boldsymbol{k}}=2\left(\tilde{s}_{0} \sigma_{0}+\tilde{t}_{3}^{x} \sigma_{x}+\tilde{t}_{3}^{x} \sigma_{y}+\tilde{t}_{3}^{z} \sigma_{z}\right) \sin \left(k_{1}-k_{2}\right) \\
+2\left(\tilde{s}_{3} \sigma_{0}+\tilde{t}_{0}^{x} \sigma_{x}+\tilde{t}_{0}^{x} \sigma_{y}+\tilde{t}_{0}^{z} \sigma_{z}\right) \cos \left(k_{1}-k_{2}\right) \\
+2\left(\tilde{s}_{0} \sigma_{0}+\tilde{t}_{3}^{z} \sigma_{x}+\tilde{t}_{3}^{x} \sigma_{y}+\tilde{t}_{3}^{x} \sigma_{z}\right) \sin \left(-k_{2}\right) \\
+2\left(\tilde{s}_{3} \sigma_{0}+\tilde{t}_{0}^{z} \sigma_{x}+\tilde{t}_{0}^{x} \sigma_{y}+\tilde{t}_{0}^{x} \sigma_{z}\right) \cos \left(-k_{2}\right) \\
+2\left(\tilde{s}_{0} \sigma_{0}+\tilde{t}_{3}^{x} \sigma_{x}+\tilde{t}_{3}^{z} \sigma_{y}+\tilde{t}_{3}^{x} \sigma_{z}\right) \sin \left(k_{1}\right) \\
+2\left(\tilde{s}_{3}+\tilde{t}_{0}^{x} \sigma_{x}+\tilde{t}_{0}^{z} \sigma_{y}+\tilde{t}_{0}^{x} \sigma_{3}\right) \cos \left(k_{1}\right), \\
B_{\boldsymbol{k}}=2\left(\tilde{s}_{0} \sigma_{0}+\tilde{t}_{3}^{x} \sigma_{x}+\tilde{t}_{3}^{z} \sigma_{y}+\tilde{t}_{3}^{x} \sigma_{z}\right) \sin \left(-k_{1}\right) \\
+ \\
+2\left(\tilde{s}_{3} \sigma_{0}+\tilde{t}_{0}^{x} \sigma_{x}+\tilde{t}_{0}^{z} \sigma_{y}+\tilde{t}_{0}^{x} \sigma_{z}\right) \cos \left(-k_{1}\right) \\
+2\left(\tilde{s}_{0} \sigma_{0}+\tilde{t}_{3}^{x} \sigma_{x}+\tilde{t}_{3}^{x} \sigma_{y}+\tilde{t}_{3}^{z} \sigma_{z}\right) \sin \left[-\left(k_{1}-k_{2}\right)\right] \\
+2\left(\tilde{s}_{3} \sigma_{0}+\tilde{t}_{0}^{x} \sigma_{x}+\tilde{t}_{0}^{x} \sigma_{y}+\tilde{t}_{0}^{z} \sigma_{z}\right) \cos \left[-\left(k_{1}-k_{2}\right)\right] \\
+2\left(\tilde{s}_{0} \sigma_{0}+\tilde{t}_{3}^{z} \sigma_{x}+\tilde{t}_{3}^{x} \sigma_{y}+\tilde{t}_{3}^{x} \sigma_{z}\right) \sin \left(k_{2}\right) \\
+2\left(\tilde{s}_{3} \sigma_{0}+\tilde{t}_{0}^{z} \sigma_{x}+\tilde{t}_{0}^{x} \sigma_{y}+\tilde{t}_{0}^{x} \sigma_{z}\right) \cos \left(k_{2}\right) .
\end{gathered}
$$

In the above scenario, we have defined the twodimensional momentum as $\mathbf{k}=k_{1} \boldsymbol{b}_{1}+k_{2} \boldsymbol{b}_{2}$, where $\boldsymbol{b}_{1}=$ $2 \pi(0,2 / \sqrt{3})$ and $\boldsymbol{b}_{2}=2 \pi(1,-1 / \sqrt{3})$ are reciprocal lattice vectors associated with Bravais lattice vectors $\boldsymbol{a}_{1}$ and $\boldsymbol{a}_{2}$, and thus we have

$$
\begin{aligned}
& k_{1}=\frac{\sqrt{3} k_{x}+3 k_{y}}{2}, \\
& k_{2}=\frac{-\sqrt{3} k_{x}+3 k_{y}}{2} .
\end{aligned}
$$

This mean-field spinon Hamiltonian (A1) is our basis to further discuss the thermal Hall effect in the spinon Fermi surface spin liquid in Sec. III.

\section{APPENDIX B: DIRAC SPIN LIQUID MEAN-FIELD HAMILTONIAN}

When we consider the Dirac spin liquid case, we instead follow the analysis in Ref. [45]. In that work, while respecting the lattice symmetry of the Kitaev- $\Gamma$ model, the meanfield Hamiltonian constructed for the Dirac spin liquid is given by

$$
\begin{aligned}
H_{\text {Dirac }}= & \sum_{\langle i j\rangle \in \alpha \beta(\gamma)}\left[C_{i}^{\dagger}\left(t_{1}^{\gamma} R_{\alpha \beta}-i t_{0}^{\gamma}+t_{2}^{\gamma} \sigma_{\gamma}\right) C_{j}+\text { H.c. }\right] \\
& +g \mu_{B} \sum_{i} C_{i}^{\dagger}\left(\frac{1}{2} \boldsymbol{B} \cdot \boldsymbol{\sigma}+\lambda\right) C_{i}+H_{0}
\end{aligned}
$$

where an $\mathrm{SU}(2)$ spinor $C_{i}=\left(f_{i, \uparrow}, f_{i, \downarrow}\right)^{T}$ and a $2 \times 2$ matrix $R_{\alpha \beta}=\frac{-i}{\sqrt{2}}\left(\sigma_{\alpha}+\sigma_{\beta}\right)$ have been introduced for convenience. The index $\alpha(\beta, \gamma)=x, y, z$ is for the nearest neighbors $\langle i j\rangle$ along three different orientations, and the other parameters are defined by $t_{1}^{\gamma}=-\frac{1}{2}|K|\left\langle C_{i}^{\dagger} R_{\alpha \beta} C_{j}\right\rangle^{*}$, $t_{0,2}^{\gamma}=-\frac{1}{8}(\Gamma-|K|)\left[\left\langle C_{i}^{\dagger} \sigma_{\alpha} R_{\alpha \beta} C_{j}\right\rangle^{*} \pm\left\langle C_{i}^{\dagger} \sigma_{\beta} R_{\alpha \beta} C_{j}\right\rangle^{*}\right]$. Here $\lambda$ is a Lagrange multiplier corresponding to the average particlenumber constraint to ensure the proper physical Hilbert space, functioning as a chemical potential, and $H_{0}$ is a constant. The $t_{1}^{\gamma}$ and $t_{2}^{\gamma}$ terms are analogous to the Rashba SOC of electrons. Rigorously speaking, all of these parameters should be determined by variational Monte Carlo calculations in which the local constraint is enforced exactly, as numerically studied by Liu and Normand [45]. Here, for convenience, we simply take several of their values to illustrate our idea of spinon thermal Hall effect in the U(1) spin liquid, but notice that when we choose these parameters, we always guarantee that the spinon (matter) field is gapless so that the system is in a deconfined phase.

\section{APPENDIX C: DUALITY PROPERTIES OF SYMMETRIC SPIN MODELS}

In spatial dimension higher than one, exactly solvable quantum Hamiltonians are rather scarce. Very interestingly, the anisotropic Hamiltonian (8) for the honeycomb lattice was found to be exactly solvable since it can be mapped to a simple Heisenberg model on all bonds simultaneously, with a hidden ferromagnet exposed by the site-dependent spin rotation that quadruples the original unit cell. This mapping has been known as the four-sublattice spin rotation trick after a work [77] for $t_{2 g}$ orbitals in a cubic environment, whose general structure was later elucidated and referred to as Klein duality in Ref. [78]. The site-dependent $\pi$ rotations of the foursublattice spin transformation connect to the Kitaev exchange through the multiplication rules of the Klein four-group.

To be concrete, for the honeycomb lattice, we consider the rotated spin operators $\tilde{\boldsymbol{S}}$ where $\tilde{\boldsymbol{S}}=\boldsymbol{S}$ for one sublattice and, depending on the particular sublattice they belong to, $\tilde{\boldsymbol{S}}$ on the remaining three sublattices differ from the original $S$ by the sign of two appropriate components [40]. Written in the rotated basis, Eq. (8) reads

$$
H_{\mathrm{ex}_{1}}=\sum_{\langle i, j\rangle \in x}-J \tilde{\boldsymbol{S}}_{i} \cdot \tilde{\boldsymbol{S}}_{j},
$$




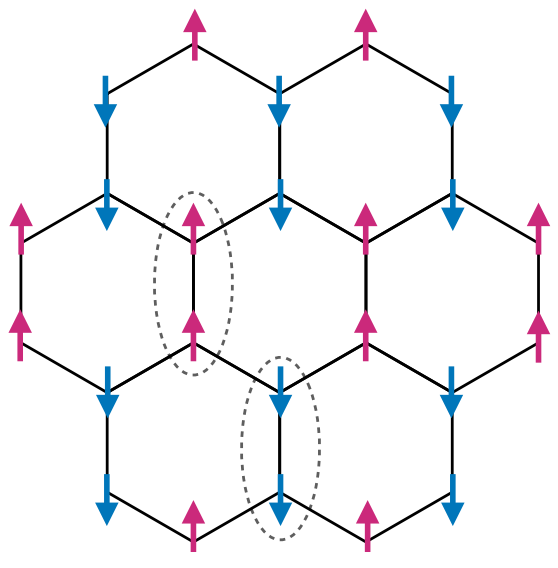

FIG. 9. Stripy antiferromagnetic state on the honeycomb lattice. Each stripe is composed of the two-site clusters (circled by the dashed gray ellipse) that reside on a given line.

with a ferromagnetic interaction. It is straightforward to obtain from Eq. (C1) that the exact ground state of Eq. (8) is a fully polarized ferromagnetic state in the rotated basis. After applying the rotation defined by the Klein duality on this magnetic order and mapping it back to the original spin basis, the resulting order is depicted in Fig. 9, which corresponds to a stripy collinear antiferromagnetic pattern of the original magnetic moments. Each stripe is composed of the two-site clusters (circled by the dashed gray ellipse in Fig. 9) that reside on a given line. Despite belonging to an antiferromagnetic type, this stripy order is fluctuation-free and would show a fully saturated antiferromagnetic order parameter [40].

Moreover, to make use of the Klein duality, the model should usually be considered to be just the pure nearestneighbor Kitaev-Heisenberg model. The duality properties generally break down when the Dzyaloshinskii-Moriya interaction and/or other further-neighbor interactions are included.

\section{APPENDIX D: ELECTRON-HOLE DOPING ASYMMETRY}

Since the proposal of the Kitaev model and Kitaev spin liquid in real materials, there has been one direction of effort in the field towards studying the effect of dopings in the relevant materials [79]. This is certainly a natural direction of thinking, on both the model level and the experimental level. One strong motivation comes from the cuprate superconductors that were often viewed as a doped Mott insulating spin liquid. The pairing already occurs in the spin liquid regime and condensed doping holes generate superconductivity. If one of the honeycomb Kitaev materials realizes the Kitaev spin liquid, doping it would probably generate topological superconductivity. This statement, however, ignores the detailed evolution of spin-orbital structure of the system under doping. There is an electron-hole doping asymmetry for such materials. If doping happens on the transition-metal ions, the electron doping would create a $d^{6}$ electron configuration that has no spin or orbital structure. In contrast, hole doping would create a $d^{4}$ electron configuration that has a reconstructed spin-orbit structure [80]. The local energy level would be a total of $J=0$ ground states, $J=1$ triplet excited states, and $J=2$ quintuplets [80]. This would create a big difference between the electron doping and the hole doping. One may compare with cuprates where an electron-hole doping asymmetry also occurs. There, electrons are doped on the $\mathrm{Cu}$ sites, while holes are doped on the $\mathrm{O}$ sites [81]. This is due to the charge-transfer nature of the insulating phase.

This electron-hole doping asymmetry and the reconstructed spin-orbital structure occur quite generically in the strong spin-orbit-coupled correlated materials with rather different electron configurations, beyond iridates or honeycomb Kitaev materials. An earlier work that considered doping $d^{4}$ Mott insulators with excitonic magnetism noted that doping constructs the spin and orbital for $d^{4}$ Mott insulators [82]. We provide a general discussion elsewhere [83].
[1] K. v. Klitzing, G. Dorda, and M. Pepper, New Method for HighAccuracy Determination of the Fine-Structure Constant Based on Quantized Hall Resistance, Phys. Rev. Lett. 45, 494 (1980).

[2] K. von Klitzing, Essay: Quantum Hall Effect and the New International System of Units, Phys. Rev. Lett. 122, 200001 (2019).

[3] D. J. Thouless, M. Kohmoto, M. P. Nightingale, and M. den Nijs, Quantized Hall Conductance in a Two-Dimensional Periodic Potential, Phys. Rev. Lett. 49, 405 (1982).

[4] D. C. Tsui, H. L. Stormer, and A. C. Gossard, Two-Dimensional Magnetotransport in the Extreme Quantum Limit, Phys. Rev. Lett. 48, 1559 (1982).

[5] R. B. Laughlin, Anomalous Quantum Hall Effect: An Incompressible Quantum Fluid with Fractionally Charged Excitations, Phys. Rev. Lett. 50, 1395 (1983).

[6] M. König, S. Wiedmann, C. Brüne, A. Roth, H. Buhmann, L. W. Molenkamp, X.-L. Qi, and S.-C. Zhang, Quantum spin hall insulator state in HgTe quantum wells, Science 318, 766 (2007).
[7] J. E. Moore, The birth of topological insulators, Nature (London) 464, 194 (2010).

[8] Y. Kasahara, T. Ohnishi, Y. Mizukami, O. Tanaka, S. Ma, K. Sugii, N. Kurita, H. Tanaka, J. Nasu, Y. Motome, T. Shibauchi, and Y. Matsuda, Majorana quantization and half-integer thermal quantum Hall effect in a Kitaev spin liquid, Nature (London) 559, 227 (2018).

[9] M. Banerjee, M. Heiblum, V. Umansky, D. E. Feldman, Y. Oreg, and A. Stern, Observation of half-integer thermal Hall conductance, Nature (London) 559, 205 (2018).

[10] X. Zhou, H. Li, J. A. Waugh, S. Parham, H.-S. Kim, J. A. Sears, A. Gomes, H.-Y. Kee, Y.-J. Kim, and D. S. Dessau, Angle-resolved photoemission study of the Kitaev candidate $\alpha-\mathrm{RuCl}_{3}$, Phys. Rev. B 94, 161106(R) (2016).

[11] K. W. Plumb, J. P. Clancy, L. J. Sandilands, V. V. Shankar, Y. F. $\mathrm{Hu}, \mathrm{K}$. S. Burch, H.-Y. Kee, and Y.-J. Kim, $\alpha-\mathrm{RuCl}_{3}$ : A spinorbit assisted Mott insulator on a honeycomb lattice, Phys. Rev. B 90, 041112(R) (2014). 
[12] L. J. Sandilands, Y. Tian, A. A. Reijnders, H.-S. Kim, K. W. Plumb, Y.-J. Kim, H.-Y. Kee, and K. S. Burch, Spin-orbit excitations and electronic structure of the putative Kitaev magnet $\alpha-\mathrm{RuCl}_{3}$, Phys. Rev. B 93, 075144 (2016).

[13] A. Koitzsch, C. Habenicht, E. Müller, M. Knupfer, B. Büchner, H. C. Kandpal, J. van den Brink, D. Nowak, A. Isaeva, and Th. Doert, $J_{\text {eff }}$ Description of the Honeycomb Mott Insulator $\alpha-\mathrm{RuCl}_{3}$, Phys. Rev. Lett. 117, 126403 (2016).

[14] I. A. Leahy, C. A. Pocs, P. E. Siegfried, D. Graf, S.-H. Do, K.-Y. Choi, B. Normand, and M. Lee, Anomalous Thermal Conductivity and Magnetic Torque Response in the Honeycomb Magnet $\alpha-\mathrm{RuCl}_{3}$, Phys. Rev. Lett. 118, 187203 (2017).

[15] S. Trebst, Kitaev materials, arXiv:1701.07056.

[16] W. Witczak-Krempa, G. Chen, Y. B. Kim, and L. Balents, Correlated quantum phenomena in the strong spin-orbit regime, Annu. Rev. Condens. Matter Phys. 5, 57 (2014).

[17] J. G. Rau, E. K.-H. Lee, and H.-Y. Kee, Spin-orbit physics giving rise to novel phases in correlated systems: Iridates and related materials, Annu. Rev. Condens. Matter Phys. 7, 195 (2016).

[18] G. Khaliullin, Orbital order and fluctuations in Mott insulators, Prog. Theor. Phys. Suppl. 160, 155 (2005).

[19] G. Chen and L. Balents, Spin-orbit effects in $\mathrm{Na}_{4} \mathrm{Ir}_{3} \mathrm{O}_{8}$ : A hyper-kagome lattice antiferromagnet, Phys. Rev. B 78, 094403 (2008).

[20] B. J. Kim, H. Jin, S. J. Moon, J.-Y. Kim, B.-G. Park, C. S. Leem, J. Yu, T. W. Noh, C. Kim, S.-J. Oh, J.-H. Park, V. Durairaj, G. Cao, and E. Rotenberg, Novel $J_{\text {eff }}=1 / 2$ Mott State Induced by Relativistic Spin-Orbit Coupling in $\mathrm{Sr}_{2} \mathrm{IrO}_{4}$, Phys. Rev. Lett. 101, 076402 (2008).

[21] G. Jackeli and G. Khaliullin, Mott Insulators in the Strong SpinOrbit Coupling Limit: From Heisenberg to a Quantum Compass and Kitaev Models, Phys. Rev. Lett. 102, 017205 (2009).

[22] A. Kitaev, Anyons in an exactly solved model and beyond, Ann. Phys. (NY) 321, 2 (2006).

[23] L. Balents, Spin liquids in frustrated magnets, Nature (London) 464, 199 (2010).

[24] L. Savary and L. Balents, Quantum spin liquids: A review, Rep. Prog. Phys. 80, 016502 (2017).

[25] Y. Vinkler-Aviv and A. Rosch, Approximately Quantized Thermal Hall Effect of Chiral Liquids Coupled to Phonons, Phys. Rev. X 8, 031032 (2018).

[26] M. Ye, G. B. Halász, L. Savary, and L. Balents, Quantization of the Thermal Hall Conductivity at Small Hall Angles, Phys. Rev. Lett. 121, 147201 (2018).

[27] Y. Kasahara, K. Sugii, T. Ohnishi, M. Shimozawa, M. Yamashita, N. Kurita, H. Tanaka, J. Nasu, Y. Motome, T. Shibauchi, and Y. Matsuda, Unusual Thermal Hall Effect in a Kitaev Spin Liquid Candidate $\alpha-\mathrm{RuCl}_{3}$, Phys. Rev. Lett. 120, 217205 (2018).

[28] R. Hentrich, M. Roslova, A. Isaeva, T. Doert, W. Brenig, B. Büchner, and C. Hess, Large thermal Hall effect in $\alpha-\mathrm{RuCl}_{3}$ : Evidence for heat transport by Kitaev-Heisenberg paramagnons, Phys. Rev. B 99, 085136 (2019).

[29] D. Watanabe, K. Sugii, M. Shimozawa, Y. Suzuki, T. Yajima, H. Ishikawa, Z. Hiroi, T. Shibauchi, Y. Matsuda, and M. Yamashita, Emergence of nontrivial magnetic excitations in a spin-liquid state of kagomé volborthite, Proc. Natl. Acad. Sci. USA 113, 8653 (2016).
[30] H. Doki, M. Akazawa, H.-Y. Lee, J. H. Han, K. Sugii, M. Shimozawa, N. Kawashima, M. Oda, H. Yoshida, and M. Yamashita, Spin Thermal Hall Conductivity of a Kagome Antiferromagnet, Phys. Rev. Lett. 121, 097203 (2018).

[31] M. Hirschberger, R. Chisnell, Y. S. Lee, and N. P. Ong, Thermal Hall Effect of Spin Excitations in a Kagome Magnet, Phys. Rev. Lett. 115, 106603 (2015).

[32] Y. H. Gao and G. Chen, Topological thermal Hall effect for topological excitations in spin liquid: Emergent Lorentz force on the spinons, arXiv:1901.01522.

[33] H. Katsura, N. Nagaosa, and P. A. Lee, Theory of the Thermal Hall Effect in Quantum Magnets, Phys. Rev. Lett. 104, 066403 (2010).

[34] C. Hickey and S. Trebst, Emergence of a field-driven U(1) spin liquid in the Kitaev honeycomb model, Nat. Commun. 10, 530 (2019).

[35] H.-C. Jiang, C.-Y. Wang, B. Huang, and Y.-M. Lu, Field induced quantum spin liquid with spinon Fermi surfaces in the Kitaev model, arXiv:1809.08247.

[36] L. Zou and Y.-C. He, Field-induced neutral Fermi surface and $\mathrm{QCD}_{3}$-Chern-Simons quantum criticalities in Kitaev materials, arXiv:1809.09091.

[37] Z. Zhu, I. Kimchi, D. N. Sheng, and L. Fu, Robust non-Abelian spin liquid and a possible intermediate phase in the antiferromagnetic Kitaev model with magnetic field, Phys. Rev. B 97, 241110(R) (2018).

[38] S. M. Winter, Y. Li, H. O. Jeschke, and R. Valentí, Challenges in design of Kitaev materials: Magnetic interactions from competing energy scales, Phys. Rev. B 93, 214431 (2016).

[39] S. M. Winter, A. A. Tsirlin, M. Daghofer, J. van den Brink, Y. Singh, P. Gegenwart, and R. Valentí, Models and materials for generalized Kitaev magnetism, J. Phys.: Condens. Matter 29, 493002 (2017).

[40] J. Chaloupka, G. Jackeli, and G. Khaliullin, Kitaev-Heisenberg Model on a Honeycomb Lattice: Possible Exotic Phases in Iridium Oxides $A_{2} \mathrm{IrO}_{3}$, Phys. Rev. Lett. 105, 027204 (2010).

[41] M. Hermanns, I. Kimchi, and J. Knolle, Physics of the Kitaev model: Fractionalization, dynamic correlations, and material connections, Annu. Rev. Condens. Matter Phys. 9, 17 (2018).

[42] G. Cao, T. F. Qi, L. Li, J. Terzic, V. S. Cao, S. J. Yuan, M. Tovar, G. Murthy, and R. K. Kaul, Evolution of magnetism in the single-crystal honeycomb iridates $\left(\mathrm{Na}_{1-x} \mathrm{Li}_{x}\right)_{2} \mathrm{IrO}_{3}$, Phys. Rev. B 88, 220414(R) (2013).

[43] I. Dzyaloshinsky, A thermodynamic theory of "weak" ferromagnetism of antiferromagnetics, J. Phys. Chem. Solids 4, 241 (1958).

[44] T. Moriya, Anisotropic superexchange interaction and weak ferromagnetism, Phys. Rev. 120, 91 (1960).

[45] Z.-X. Liu and B. Normand, Dirac and Chiral Quantum Spin Liquids on the Honeycomb Lattice in a Magnetic Field, Phys. Rev. Lett. 120, 187201 (2018).

[46] X.-G. Wen, Quantum orders and symmetric spin liquids, Phys. Rev. B 65, 165113 (2002).

[47] D. Sen and R. Chitra, Large- $U$ limit of a Hubbard model in a magnetic field: Chiral spin interactions and paramagnetism, Phys. Rev. B 51, 1922 (1995).

[48] O. I. Motrunich, Orbital magnetic field effects in spin liquid with spinon Fermi sea: Possible application to $\kappa-(\mathrm{ET})_{2} \mathrm{Cu}_{2}(\mathrm{CN})_{3}$, Phys. Rev. B 73, 155115 (2006). 
[49] X. G. Wen, F. Wilczek, and A. Zee, Chiral spin states and superconductivity, Phys. Rev. B 39, 11413 (1989).

[50] P. A. Lee and N. Nagaosa, Gauge theory of the normal state of high- $T_{c}$ superconductors, Phys. Rev. B 46, 5621 (1992).

[51] P. A. Lee, N. Nagaosa, and X.-G. Wen, Doping a Mott insulator: Physics of high-temperature superconductivity, Rev. Mod. Phys. 78, 17 (2006).

[52] A. M. Polyakov, Quark confinement and topology of gauge theories, Nucl. Phys. B 120, 429 (1977).

[53] J. M. Luttinger, Theory of thermal transport coefficients, Phys. Rev. 135, A1505 (1964).

[54] T. Qin, Q. Niu, and J. Shi, Energy Magnetization and the Thermal Hall Effect, Phys. Rev. Lett. 107, 236601 (2011).

[55] F. J. Burnell and C. Nayak, SU(2) slave fermion solution of the Kitaev honeycomb lattice model, Phys. Rev. B 84, 125125 (2011).

[56] P. Zanardi and N. Paunković, Ground state overlap and quantum phase transitions, Phys. Rev. E 74, 031123 (2006).

[57] V. Kalmeyer and R. B. Laughlin, Equivalence of the Resonating-Valence-Bond and Fractional Quantum Hall States, Phys. Rev. Lett. 59, 2095 (1987).

[58] Y.-D. Li, X. Yang, Y. Zhou, and G. Chen, Non-Kitaev spin liquids in Kitaev materials, Phys. Rev. B 99, 205119 (2019).

[59] K. Kitagawa, T. Takayama, Y. Matsumoto, A. Kato, R. Takano, Y. Kishimoto, S. Bette, R. Dinnebier, G. Jackeli, and H. Takagi, A spin-orbital-entangled quantum liquid on a honeycomb lattice, Nature (London) 554, 341 (2018).

[60] Y. S. Choi, C. H. Lee, S. Lee, S. Yoon, W.-J. Lee, J. Park, A. Ali, Y. Singh, J.-C. Orain, G. Kim, J.-S. Rhyee, W.-T. Chen, F. Chou, and K.-Y. Choi, Exotic Low-Energy Excitations Emergent in the Random Kitaev Magnet $\mathrm{Cu}_{2} \mathrm{IrO}_{3}$, Phys. Rev. Lett. 122, 167202 (2019).

[61] K. Slagle, W. Choi, L. E. Chern, and Y. B. Kim, Theory of a quantum spin liquid in the hydrogen-intercalated honeycomb iridate $\mathrm{H}_{3} \mathrm{LiIr}_{2} \mathrm{O}_{6}$, Phys. Rev. B 97, 115159 (2018).

[62] R. Yadav, R. Ray, M. S. Eldeeb, S. Nishimoto, L. Hozoi, and J. van den Brink, Strong Effect of Hydrogen Order on Magnetic Kitaev Interactions in $\mathrm{H}_{3} \mathrm{LiIr}_{2} \mathrm{O}_{6}$, Phys. Rev. Lett. 121, 197203 (2018).

[63] Y. Li, S. M. Winter, and R. Valentí, Role of Hydrogen in the Spin-Orbital-Entangled Quantum Liquid Candidate $\mathrm{H}_{3} \mathrm{LiIr}_{2} \mathrm{O}_{6}$, Phys. Rev. Lett. 121, 247202 (2018).

[64] J. Knolle, R. Moessner, and N. B. Perkins, Bond-Disordered Spin Liquid and the Honeycomb Iridate $\mathrm{H}_{3} \operatorname{LiIr}_{2} \mathrm{O}_{6}$ : Abundant Low-Energy Density of States from Random Majorana Hopping, Phys. Rev. Lett. 122, 047202 (2019).

[65] I. Kimchi, J. P. Sheckelton, T. M. McQueen, and P. A. Lee, Scaling and data collapse from local moments in frustrated disordered quantum spin systems, Nat. Commun. 9, 4367 (2018).
[66] D. S. Fisher, Random Transverse Field Ising Spin Chains, Phys. Rev. Lett. 69, 534 (1992).

[67] D. S. Fisher, Random antiferromagnetic quantum spin chains, Phys. Rev. B 50, 3799 (1994).

[68] D. S. Fisher, Critical behavior of random transverse-field Ising spin chains, Phys. Rev. B 51, 6411 (1995).

[69] E. Altman, Y. Kafri, A. Polkovnikov, and G. Refael, Insulating Phases and Superfluid-Insulator Transition of Disordered Boson Chains, Phys. Rev. Lett. 100, 170402 (2008).

[70] O. Motrunich, S.-C. Mau, D. A. Huse, and D. S. Fisher, Infiniterandomness quantum Ising critical fixed points, Phys. Rev. B 61, 1160 (2000).

[71] O. Motrunich, K. Damle, and D. A. Huse, Particle-hole symmetric localization in two dimensions, Phys. Rev. B 65, 064206 (2002).

[72] C. R. Laumann, D. A. Huse, A. W. W. Ludwig, G. Refael, S. Trebst, and M. Troyer, Strong-disorder renormalization for interacting non-Abelian anyon systems in two dimensions, Phys. Rev. B 85, 224201 (2012).

[73] J. Cookmeyer and J. E. Moore, Spin-wave analysis of the lowtemperature thermal Hall effect in the candidate Kitaev spin liquid $\alpha-\mathrm{RuCl}_{3}$, Phys. Rev. B 98, 060412(R) (2018).

[74] Y.-D. Li and G. Chen, Detecting spin fractionalization in a spinon fermi surface spin liquid, Phys. Rev. B 96, 075105 (2017).

[75] L. Balents and O. A. Starykh, Spinon waves in magnetized spin liquids, arXiv:1904.02117.

[76] P. Rao and I. Sodemann, Cyclotron resonance inside the Mott gap: A fingerprint of emergent neutral fermions, arXiv:1905.08271.

[77] G. Khaliullin and S. Okamoto, Quantum Behavior of Orbitals in Ferromagnetic Titanates: Novel Orderings and Excitations, Phys. Rev. Lett. 89, 167201 (2002).

[78] I. Kimchi and A. Vishwanath, Kitaev-Heisenberg models for iridates on the triangular, hyperkagome, kagome, fcc, and pyrochlore lattices, Phys. Rev. B 89, 014414 (2014).

[79] Y.-Z. You, I. Kimchi, and A. Vishwanath, Doping a spin-orbit Mott insulator: Topological superconductivity from the KitaevHeisenberg model and possible application to $\left(\mathrm{Na}_{2} / \mathrm{Li}_{2}\right) \mathrm{IrO}_{3}$, Phys. Rev. B 86, 085145 (2012).

[80] G. Chen and L. Balents, Spin-orbit coupling in $d^{2}$ ordered double perovskites, Phys. Rev. B 84, 094420 (2011).

[81] M. Randeria, R. Sensarma, N. Trivedi, and F.-C. Zhang, Particle-Hole Asymmetry in Doped Mott Insulators: Implications for Tunneling and Photoemission Spectroscopies, Phys. Rev. Lett. 95, 137001 (2005).

[82] J. Chaloupka and G. Khaliullin, Doping-Induced Ferromagnetism and Possible Triplet Pairing in $d^{4}$ Mott Insulators, Phys. Rev. Lett. 116, 017203 (2016).

[83] G. Chen and A. Paramekanti (unpublished). 